\section{$\underset{\substack{\text { hommes } \\ \text { \& migrations }}}{ }$}

\section{Hommes \& migrations}

Revue française de référence sur les dynamiques

migratoires

$1309 \mid 2015$

Le $3 e$ âge des migrants

\title{
Les familles face à la maladie d'Alzheimer
}

Le parcours du combattant des aidants

Jenny Duchier et Jean Mantovani

\section{OpenEdition}

1 Journals

\section{Édition électronique}

URL : http://journals.openedition.org/hommesmigrations/3082

DOI : 10.4000/hommesmigrations.3082

ISSN : 2262-3353

Éditeur

Musée national de l'histoire de l'immigration

Édition imprimée

Date de publication : 1 janvier 2015

Pagination : 87-94

ISBN : 978-2-919040-30-8

ISSN : 1142-852X

Référence électronique

Jenny Duchier et Jean Mantovani, «Les familles face à la maladie d'Alzheimer », Hommes \& migrations [En ligne], 1309 | 2015, mis en ligne le 01 janvier 2017, consulté le 19 avril 2019. URL : http:// journals.openedition.org/hommesmigrations/3082 ; DOI : 10.4000/hommesmigrations.3082 


\title{
LES FAMILLES FACE À LA MALADIE D'ALZHEIMER
} LE PARCOURS DU COMBATTANT DES AIDANTS

par JENNY DUCHIER, ethnologue, ingénieur d'études, unité Inserm (UMR 1027), université Paul-Sabatier, Toulouse, et JEAN MANTOVANI, sociologue, chargé de recherche, Observatoire régional de la santé de Midi-Pyrénées (ORSMIP) et unité Inserm (UMR 1027), université Paul-Sabatier, Toulouse.

\author{
Face aux pathologies de type Alzheimer, les migrants \\ vieillissants originaires des pays du Maghreb et, à un moindre \\ degré, leurs descendants continuent de passer à côté \\ des dispositifs publics de soin à domicile. Affaire familiale, \\ les troubles de la cognition et du comportement des plus vieux \\ sont pris en charge par les proches, souvent jusqu'à \\ l'épuisement. Des représentations erronées de la maladie, \\ le sentiment de honte qu'elle suscite font se renfermer \\ les familles concernées. Et le chez-soi protégé risque de devenir \\ pour le malade un espace de confinement.
}

Les troubles de la cognition et du comportement de type Alzheimer soulèvent un ensemble de problématiques que la gérontologie sociale, comme domaine de recherche et de pratiques professionnelles, maitrise relativement, même s'il faut regretter que les efforts consacrés à mieux comprendre les conséquences de la pathologie sur les personnes et leur entourage et à adapter les moyens en conséquence restent insuffisants. On sait, par exemple, ce qu'il en est généralement des sentiments de honte et de culpabilité que ce type de pathologie induit pour les patients et les proches, des processus de repli et d'enfermement, de la réticence des aidants familiaux à faire appel à un support professionnel, des fréquents renoncements aux soins, du "fardeau" des aidants, le plus souvent des aidantes, mais aussi des conjoints... On sait aussi à quel point l'apparition de troubles du comportement met en péril le maintien à domicile des personnes atteintes... Même si des changements se manifestent lentement au sujet de l'approche de ces troubles et des postures d'acteurs, la stigmatisation 
des personnes et des troubles demeure très forte, à l'instar de la plupart des pathologies mentales.

L'objet de cet article est de rappeler en quoi les répercussions de la maladie sur les malades et leur entourage informel provoquent un ensemble de difficultés spécifiques dans les familles issues de l'immigration maghrébine de l'après-Seconde Guerre mondiale et à quel point les patients et ces familles soumis aux aléas cumulés de la pauvreté, de l'altérité, de la vieillesse et de la maladie apparaissent en déficit d'accès aux services et aux soins et à la citoyenneté...

Nous nous appuyons en cela sur les résultats de la recherche Migrants âgés et troubles cognitifs $(\mathrm{MATC})^{1}$, qui a donné lieu à un rapport en juin 2014. L'investigation de terrain, réalisée essentiellement par entretiens enregistrés et retranscrits, s'est attachée à élaborer des monographies de situations familiales impliquant au moins une personne âgée atteinte de troubles de type Alzheimer, dont nous avons pu prendre connaissance soit en structure hospitalière, soit à domicile, grâce à différents médiateurs. Le volet principal de la recherche s'est enrichi d'entretiens auprès de divers professionnels en grande partie issus de l'immigration, interrogés au sujet de leurs pratiques et points de vue sur les situations de migrants âgés malades et sur leurs conditions de prise en charge soignante ou aidante.

\section{Les migrants vieillissants face au durcissement des politiques migratoires}

Les résultats de ce travail concernent une génération de migrants vieillissants originaires des pays du Maghreb et leur entourage actuel. Cette génération des migrants des Trente Glorieuses a connu un contexte historique plutôt favorable. Mais il est également marqué par le basculement des années 1970 dans une société de précarisation de masse et de renversement des politiques de l'immigration. C'est, par ailleurs, au début des années 1960 qu'il faut situer l'avènement des politiques gérontologiques du "maintien des personnes âgées à domicile2", avec le développement des services idoines, en un temps où s'accroissait paradoxalement l'offre d'hébergement en institution et de placement hospitalier de longue durée. Mais le contexte des Trente Glorieuses et des décennies suivantes se caractérise aussi par la permanence, sinon l'accroissement des inégalités sociales de statut et un isolement grandissant des vieux les plus pauvres.

Une autre composante importante de cette période tient au fait migratoire : le besoin de main-d'œuvre était très important en métropole après la Deuxième Guerre mondiale. Des politiques volontaristes, visant à instituer ou du moins à faciliter l'arrivée de travailleurs étrangers, voient le jour. Ces politiques ciblent en particulier la jeune maind'œuvre originaire des pays du Maghreb, en particulier à l'orée des années 1960 et à la fin de la guerre d'Algérie, en un temps où le potentiel des pays du sud de l’Europe apparaît déclinant. Elles s'attachent également à aider à l'installation et à la régularisation en France de jeunes originaires des pays du Maghreb entrés en dehors du monopole d'État sur l'introduction de la main-d'œuvre étrangère dans le pays... jusqu'à leur remise en cause radicale à partir de 1974 (fin des Trente Glorieuses) et surtout avec les dispositions Stoléru de 1977-1978 : abrogation des dispositions antérieures sur l'immigration organisée, aide au "retour volontaire", puis mesures d'expulsion des immigrés sans contrat de travail considérés comme "clandestins", qui épargnent en partie l'immigration européenne mais visent tout particulièrement les personnes originaires des États du Maghreb.

Ce retour historique résume le renversement qu'ont subi les anciens de l'immigration maghrébine des Trente Glorieuses et leur sentiment d'être devenus 
Chambre d' Azib Benterki, 77 ans, Algérien et ancien syndicaliste. C'est l'un des plus anciens du foyer Adoma "Les Grésillons", à Gennevilliers, $1^{\text {er }}$ avril $2015 \odot$ Camille Millerand

la cible de politiques restrictives, sinon répressives, dans lesquelles leurs droits ont été, au moins symboliquement, remis en cause au plus haut niveau des institutions nationales. Parmi ceux de ces anciens "jeunes travailleurs immigrés" qui sont restés attachés au pays d'accueil, les primo-arrivants ont, dans leur majorité, fondé une famille et se sont fortement "enracinés" en France. Mais nombreuses sont les personnes et les familles qui ont continué à entretenir des relations étroites avec des proches restés "au pays", certaines pratiquant différentes formes de résidence en alternance entre leur "pays" d'origine et leur lieu de vie et de travail "ici". Dans tous les cas, le vieillissement des immigrés maghrébins de la première génération soulève des questions jusque-là bien peu considérées en termes de santé publique. Pourtant, un nombre croissant de migrants vieillissants souffrent aujourd'hui des troubles considérés.

\section{Identifier les causes du renoncement aux soins}

Le questionnement principal se pose d'abord en termes de non-recours à la prévention et aux soins, et surtout de renoncement ou de refus de faire appel aux dispositifs existants. Avant d'aborder 
nos propres résultats de recherche, on renverra aux données comparatives récentes de l'Institut national d'études démographiques (Ined $\left.{ }^{4}\right)$ construites à partir d'indicateurs, toutes pathologies confondues, de non-recours et de renoncement aux soins. Les résultats établissent notamment que ces situations sont deux à trois fois plus nombreuses parmi les immigrés hommes d'Algérie, du Maroc et de Tunisie que parmi les non-immigrés.

Il ressort de notre recherche qualitative qu'un large faisceau de facteurs participe au non-recours, au renoncement, et plus largement à un fort retrait des patients dans la sphère familiale et, ainsi, à des recours souvent très tardifs. En témoignent les difficultés rencontrées au début de la recherche pour entrer en contact avec les malades et les familles.

La plupart des services de proximité contactés, y compris dans des espaces d'habitat à forte concentration de familles issues de l'immigration maghrébine, ne comptaient guère d'usagers de cette catégorie et étaient bien en peine de faire état d'une demande. Il en est allé de même, à un moindre degré, avec les services départementaux en charge de la gestion de l'allocation personnalisée d'autonomie (APA), ou encore avec la médecine générale et les services hospitaliers. Ces derniers ont fourni le principal de notre corpus d'enquête, mais essentiellement auprès de patients souffrant de troubles de type Alzheimer à un stade très évolué de la pathologie. Les migrants vieillissants originaires des pays du Maghreb, ainsi que leurs descendants, à un moindre degré, continuent de passer à côté des politiques engagées. Leurs conditions d'accès aux soins, au care et aux services restent très déficitaires, en particulier aux stades les moins évolués de la pathologie. Les troubles de la cognition et du comportement des plus vieux restent une affaire essentiellement familiale, qui ne prend forme dans le domaine public que lorsque les moyens du soutien familial arrivent à épuisement.

\section{Sens de la famille et repli sur soi}

Les migrants âgés et leur entourage expriment le plus souvent une forte réticence (surtout les personnes âgées, mais souvent également les aidants familiaux) à faire appel à une intervention extérieure. Cette réticence confine parfois au renoncement au soutien et aux soins professionnels, surtout pour ce qui a trait au corps et aux soins corporels. Y participe le sentiment, souvent très marqué dans le contexte de grandes fratries de descendants, qu'il est du devoir des enfants d'apporter seuls le soutien quotidien et les soins corporels devenus indispensables pour sauvegarder la dignité, l'intégrité de leurs parents malades et dépendants. Toutes les recherches antérieures ont montré que ce sens du devoir des enfants vis-à-vis de leurs parents reste très largement partagé, y compris dans les familles non issues de l'immigration ${ }^{5}$, mais il prend dans ce cas une dimension de plus grande exclusivité dans le renversement des rôles parents/enfants : aux yeux des enfants, les parents sont reconnus pour avoir apporté toute leur attention à protéger et soigner les enfants, déjà dans des conditions d'autonomisation et de fort repli sur l'espace familial. Ils ont porté plus particulièrement leur affection sur un ou plusieurs enfants "désignés", le plus souvent une ou plusieurs des filles, qui se sont plus ou moins destinée(s) assez tôt à assurer plus tard la gestion de la situation de leur(s) parent(s) fragilisé(s). L'espace du foyer des parents, ou celui de la cohabitation parents/enfants, est considéré comme l'espace du chez-soi protégé, de l'intimité, de la pudeur et de la discrétion, dans lequel l'intrusion d'opérateurs non "familialisés" reste confinée aux situations de crise ou aux situations limites. 


\section{Faire face aux troubles de type Alzheimer}

L'apparition de troubles légers de la cognition est le plus souvent interprétée comme la manifestation d'un vieillissement non pathologique, les relations privilégiées entre parents et enfants restant préservées. Il faut du temps avant que ne se construise parmi les proches une représentation des symptômes vécus comme relevant de la maladie. Pendant cette période de construction, il s'agit d'abord d'éloigner le spectre de la maladie d'Alzheimer, considérée comme synonyme à la fois de folie et de vieillesse dégradée et dégradante, qu'il serait très difficile d'afficher en public. Il en va ainsi d'un double risque de stigmatisation et de perte de statut pour les parents et leurs enfants. Lorsque les troubles du comportement du malade (agressivité ou déviance) apparaissent, ils deviennent incompatibles avec les codes sociaux des proches et de leurs voisins. Lattitude des proches devient paradoxale : c'est souvent devant ce type de situation que les aidants familiaux se résolvent à faire appel aux soignants, et d'abord au médecin traitant, le plus accessible a priori. L'inadaptation chronique de l'offre d'hébergement et plus largement de soutien extra familial, mais aussi celle de l'offre hospitalière de soins de moyen séjour et de longue durée comme des outils de l'évaluation gériatrique standardisée et des services de soins spécialisés, confirment les familles d'origine immigrée maghrébine dans leur refus du placement en EHPAD ${ }^{6}$. Les acteurs du soin, comme ceux des services d'aide à domicile, se bornent souvent à considérer la barrière de la langue, l'altérité des cultures, mesurée notamment sur le plan des étiologies profanes de la maladie ou à travers le filtre des valeurs de la famille et du repli sur l'entre-soi communautaire...
Selon le volet d'une enquête auprès des professionnels, une bonne part des soignants considèrent quétant donné les limitations dans lesquelles se situent les thérapeutiques de la maladie d'Alzheimer, les difficultés de communication avec les malades et leurs descendants, le déficit d'interprétariat, l'introversion des familles immigrées et la proximité active des enfants..., mieux vaut que le soutien aux patients âgés revienne, aussi longtemps que possible, à leurs enfants, c'est-à-dire le plus souvent à leur(s) fille(s) désignée(s). La boucle de l'enfermement des vieux immigrés malades et de leurs proches se trouve ainsi refermée. Encore faut-il évoquer les limites d'un "GIRage" qui méconnait assez largement les troubles de type Alzheimer, au point d'accorder deux heures d'aide ménagère par semaine aux familles qui assurent elles-mêmes le ménage mais ont besoin de tout autre chose en termes d'accompagnement. ${ }^{8} \quad$ Ainsi peut-on résumer le contexte dans lequel s'est déroulée notre recherche, et le proces-

L'apparition de troubles légers de la cognition est le plus souvent interprétée comme la manifestation d'un vieillissement non pathologique, les relations privilégiées entre parents et enfants restant préservées. sus qui a fait que l'essentiel de notre corpus a été constitué à partir de situations de personnes âgées en fin de vie dont nous avons rencontré l'entourage familial et professionnel dans les services de type unités de soins de longue durée (USLD), auxquels les aidants familiaux avaient fait appel en désespoir de cause.

\section{Un engagement total qui confine souvent à l'épuisement}

Il faut évoquer ici quelques situations vécues de soutien familial parmi les plus significatives. Mme F. a 52 ans lors de l'enquête. Elle dit elle-même qu'elle a 
"atteint une limite" : "J'ai été sans relâche en fait, c'est un parcours vraiment du combattant." Trois ans après le début des troubles graves de son père, on lui diagnostique un cancer du sein. Elle doit être opérée. Quelques jours avant l'intervention, elle disait encore: "Tout en étant malade, je m'occupais de mon père, jusqu'au (moment) où j'allais faire l'ablation." Son père est hospitalisé le jour de son opération et "il n'est pas retourné à domicile... je ne pouvais plus m'en occuper". D'autres facteurs ont participé au "fardeau" de la fille aidante, et notamment le difficile arbitrage dans lequel elle s'est trouvée contrainte entre le soutien à son père et le soutien à ses enfants: "Je me disais aussi, pourquoi je ne moccupais que de lui, parce que, bon, lui il est malade, ce n'est pas pareil, mes enfants ils sont grands, je pense qu'ils sont assez grands pour s'assumer."

Mme L. est la dernière fille d'une fratrie de sept enfants. Elle s'occupe de son père et de sa mère, 94 ans et 82 ans, cette dernière considérée comme la plus malade du fait de son trouble psychique. Le couple âgé est depuis peu bénéficiaire de l'allocation personnalisée d'autonomie (APA). C'est Mme L., âgée de 42 ans, qui assume l'essentiel comme salariée familiale depuis sept ans. Elle présente sa démarche comme spontanée et volontaire, mais elle se sent assez peu reconnue par les autres membres de la fratrie : "Au fur et à mesure, je me rendais compte que ça faisait plus de responsabilités. Mes frères et sceurs viennent, mais c'est moi qui m'en occupe parce que j'ai commencé par prendre en charge le côté médical en fait et, maintenant, ils se sont habitués. C'est moi qui m'en charge, au fur et à mesure c'est devenu naturel. Je me suis proposée. C'est vrai qu'à cette époque tout le monde était plus ou moins occupé. On disait qu'on allait essayer de faire un tour de rôle mais non, personne ne s'est rendu disponible finalement et c'est vrai que c'est pesant. J'aurais voulu que quelqu'un d'autre s'en occupe à ma place."

Mme M. est l'une des filles qui assure l'essentiel de l'aide sur fond de grand sens du devoir familial et de refus absolu de ce qui pourrait être considéré comme un abandon des parents : “C'est pas possible! On ne peut pas! Elle était là pour nous, on ne voit pas pourquoi on ne serait pas là pour elle, elle a eu 8 enfants." Mais tous les membres de la fratrie ne sont pas aussi disponibles, et la sœur aînée est dans le déni de la pathologie de la mère. La fille "désignée" pour coordonner le dispositif familial dit: "Je n'ai pas le choix, je ne peux pas la laisser, on ne sait jamais... Elle compte davantage sur moi... on essaie de se soutenir." Un autre fils cohabite avec sa mère : "J'essaie de gérer", mais l'aidante relève aussi combien la prise en charge est devenue lourde pour la fille aînée. C'est cet engagement de tous les jours qui l'a poussée à solliciter une aide professionnelle extérieure.

M. A. était retourné en Algérie au moment de la retraite afin de vivre auprès de sa famille élargie. Mais il va connaître assez rapidement des problèmes de santé mal définis. Nous le rencontrons en milieu hospitalier. Deux de ses enfants, venus séjourner en France, ont organisé son retour dans la région parisienne en concertation avec le reste de la fratrie, en quête de soins auprès des gériatres français. En Algérie, dit le fils, "les moyens sont catastrophiques. Sincèrement, si on trouve les moyens là-bas, adaptables à sa maladie, je préfère rester là-bas. Mais il a son assurance ici, et ça se dégrade." Les enfants, comme la famille tout entière, ne veulent pas entendre parler de pathologie de type Alzheimer et s'accrochent à l'idée que leur père a été victime d'accidents vasculaires cérébraux. Mais son déclin cognitif est déjà bien engagé, et si les enfants envisagent encore de retourner au plus vite dans leur pays, la situation est des plus incertaines et le soutien informel atteint ses limites.

\section{Représentations culturelles et discriminations}

Cette recherche avait pour objectif de comprendre le poids de la dimension culturelle dans l'attitude des chibanis et de leurs enfants face aux troubles de type Alzheimer. Car un statut particulier pèse sur 
les personnes et les familles identifiées à la figure du travailleur immigré d’origine maghrébine. Dans le contexte plus ou moins marqué de stigmatisation et de discrimination qu'ils ont connu à leur arrivée, les cadres familiaux, comme les relations entre pairs, ont constitué des supports et une ressource essentiels pour l'autonomisation de chacun. Ainsi, l'accès à l'autonomie des individus tient souvent beaucoup aux collectifs constitués.

Si leurs enfants se montrent également très attachés aux valeurs de la famille, c'est avant tout parce que celle-ci est aujourd'hui dépositaire de valeurs essentielles. C'est donc moins en référence à une culture des racines et des héritages quà une culture de l'indépendance, de l'autosuffisance et de la solidarité. Le fait que les descendants continuent, comme c'est le cas face à la vieillesse et à la maladie de leurs parents, à cultiver la différence entre ce qui se fait "chez nous" et ce qui se fait "chez vous" interroge sans doute les dissemblances culturelles, mais davantage la persistance d'un sentiment d'altérité : "Chez nous, on n'abandonne pas les parents", "les envoyer en maison de retraite, ça ne se fait pas", "cest notre devoir de nous en occuper"... On sait ce qu'il en est des limites de ces représentations, le sens du devoir familial restant le plus souvent très fort, $\mathrm{y}$ compris parmi les non-migrants les plus pauvres et les moins autonomes, et même dans la majorité des familles plus favorisées.

\section{Conclusion}

Il existe bien peu de raisons en termes de gérontologie sociale de différencier au nom de la culture le "prendre soin d'un proche âgép" chez les familles d'origine maghrébine confrontées aux pathologies de type Alzheimer. Surtout, rien ne justifie de renvoyer la condition des familles d'origine maghrébine à un essentialisme culturaliste, qui aurait pour effet d'enfermer plus encore les aidants familiaux à leur propre responsabilité individuelle et collective. Il faut plus que jamais considérer la culture des personnes, des familles et groupes sociaux issus de limmigration comme relevant des interactions sociétales dans lesquelles se trouvent prises les familles issues de l'immigration. Aujourd'hui, les immigrés maghrébins se trouvent confrontés à des problématiques associées il n'y pas si longtemps à d'autres migrants et non-migrants : Italiens, Espagnols, rapatriés d'Algérie..., et plus largement à toutes les composantes sociales les plus populaires. Le décalage n'en existe pas moins : les valeurs du familialisme sont celles des familles pauvres qui ne tablent guère sur d'autres recours que sur l'autosupport "informel". Face aux troubles de type Alzheimer de leur(s) parent(s) âgés, la culture se manifeste essentiellement par la non-demande, le non-recours et le renoncement à l'aide des services existants, sous prétexte que les troubles de la vieillesse relèvent d'abord de la sphère privée. Ce décalage s'inscrit dans le temps et dans l'espace ${ }^{10}$ des personnes issues de la migration maghrébine. Il questionne aussi les institutions en France, l'état des organisations constituées au service du "maintien à domicile" des plus vieux, notamment les dispositifs en place dans leurs capacités à considérer la révision des repères identitaires que suscite la maladie, et les conséquences sur la santé des proches aidants.

Deux questions majeures s'imposent concernant la situation des familles d'origine des pays du Maghreb. La première est celle de l'enfermement des vieux dans l'univers familial. Si rien dans notre recherche ne nous permet de parler de maltraitance ou de négligence envers les parents âgés ${ }^{11}$, il est inévitable que des situations de ce genre existent ${ }^{12}$. La seconde concerne le "fardeau" qui pèse sur les aidantes (plus rarement sur des fils aidants), ces "combattantes" qui justifient le titre de cet article. C'est le plus souvent leur épuisement total qui fait a posteriori de leur sort une question publique. 
À ce jour, la situation des familles d'origine maghrébine confrontées aux pathologies de type Alzheimer de leurs ascendants réactive une vieille question relative aux inégalités sociales de santé, inégalités d'accès aux droits, à la citoyenneté, aux institutions, aux soins et aux services. Si elle ne met pas en cause les droits ouverts, elle interroge les capacités de médiation, d'interrelation et de fraternité de l'action publique.

\section{Bibliographie}

- Attias-Donfut Claudine, Gallou Rémi, "Impact des cultures d'origine sur les pratiques d'entraide familiale. Représentation de la solidarité familiale pour les immigrés âgés", in Informations sociales, vol. 6, n ${ }^{\circ}$ 134, 2006, pp. 86-97.

- Attias-Donfut Claudine, L’Enracinement. Enquête sur le vieillissement des immigrés, Paris, Armand Colin, 2007.

- Bartkowiak Nadège, L’Accueil des immigrés vieillissants en institution. Réflexions-actions autour de l'accueil en établissement d'hébergement pour personnes âgées dépendantes, Rennes, Presses de l'EHESP, 2008.

- Coudin Geneviève, "La réticence des aidants familiaux à recourir aux services gérontologiques : une approche psychosociale", in Psychol NeuroPsychiatr Vieil, vol. 2, $\mathrm{n}^{\circ} 4$, 2004, pp. 285-296.

- Després Caroline, Dourgnon Paul, Fantin Romain, Jusot Florence, "Le renoncement aux soins : une approche socio-anthropologique", Questions d'économie de la santé, n¹69, IRDES, 2011, pp. 1-7.

- Duguet Anne-Marie, Duchier Jenny, Boyer-Bévière Bénédicte, "Maghrébins âgés dépendants, les facteurs culturels sont-ils un frein à l'accès aux dispositifs d'aide en France?", in Fabrice Gzil, Emmanuel Hirsch (dir.), Alzheimer, éthique et société, Toulouse, Eres, 2012, pp. 472-485.
- Jacquat Denis, Bachelay Alexis, "Rapport d'information au nom de la Mission d'information sur les immigrés âgés", n 1214, 2013.

http://www.assemblee-nationale.fr/14/ rap-info/i1214.asp

- Jacquet Isabelle, La Vieillesse au Maroc, Louvain, Academia-Bruylant, 2009.

- Nasraoui Mustapha, La Vieillesse dans la société tunisienne, Paris, L'Harmattan, 2003.

- Observatoire régional de la santé de MidiPyrénées, "Les personnes immigrées vieillissantes en Midi-Pyrénées, conditions d'accès aux soins et aux services", rapport, 2011. http://www.orsmip.org/tlc/documents/ migrantsjanv2013.pdf

- Paquet Mario, "La réticence familiale à recourir au soutien formel : un obstacle à la prévention de l'épuisement des personnes-soutiens de personnes âgées dépendantes", in Nouvelles pratiques sociales, vol. 10, $\mathrm{n}^{\circ}$ 1, 1997, pp. 111-124.

- Pitaud Philippe, Exclusion, maladie d'Alzheimer et troubles apparentés. Le vécu des aidants, Paris, Eres, 2006.

- Rafaï Kadija, Mantovani Jean, Duchier Jenny, Gayral-Taminh Martine, "Le vieillissement des migrants, situation en MidiPyrénées", rapport de l'Observatoire régional de la santé de Midi-Pyrénées, 2007.

- Samaoli Omar, Lindblad Peter, Amstrup Kirsten, Patel Naina, Mirza Naheed, Vieillesse, démence et immigration. Pour une prise en charge adaptée des personnes âgées migrantes en France, au Danemark et au Royaume-Uni, Paris, L'Harmattan, 2000.

- Sayad Abdelmalek, "Vieillir... dans l'immigration", in Migrations Santé, n 99/100, 1999, pp. 7-22. 